$\Rightarrow$

\section{Genomics toolkit for UPEC}

DOI:

10.1038/nrmicro1698

URLs

UT189

http://www.ncbi.nlm.nih.gov/

entrez/query.fcgi?db=genome

prj $\delta \mathrm{cmd}=$ Retrieve $\varepsilon$ dopt $=$ Over

viewslist_uids=16259

MG1655

http://www.ncbi.nlm.nih.gov/ entrez/query.fcgi?db=genome $\varepsilon c m d=$ Retrieve\&dopt=Overvie wElist_uids $=115$

The ability to alternate between different environments is a

valuable asset for would-be opportunistic pathogens. Uropathogenic Escherichia coli (UPEC) can make the transition from life as a benign commensal in the colon to life as a pathogen inside epithelial cells in the bladder. Reigstad and colleagues have developed functional genomics tools to probe the interactions between

cultures of MG1655 and UTI89 indicated that the physiology of these strains was similar in aerobic and anaerobic conditions. In the caeca of mice, however, it was a different story - compared with MG1655, UTI89 significantly upregulates transcription of 67 genes, including those involved in ferrous iron transport and the transport of iron-siderophore complexes across UPEC and the host.

Urinary tract infections (UTI) range from mild cystitis to painful pyelonephritis, and although not typically fatal, are common and often recur. More than $80 \%$ of UTIs are caused by UPEC, a species which is usually present in the colonic microbiota, but which can gain access to the urethra and bladder through the periurethra and vagina. Once in the bladder, bacteria invade epithelial umbrella cells and form intracellular bacterial communities (IBCs) in the cytoplasm that protrude out of the epithelia to form structures named pods. IBCs are protected from the immune response and antibiotics. Cells in IBCs can dissociate, sloughing off bacteria that infect cells anew, thereby continuing the cycle of infection and, in some hosts, producing recurrent bouts of acute disease.

The UPEC strain UTI89, which was isolated from a cystitis sufferer, was sequenced in 2006, and the authors have now exploited this resource to implement a functional genomics strategy to investigate UPEC pathogenesis. UTI89 shares $70 \%$ of its ORFs with the benign E. coli strain MG1655, and this strain served as a comparison in transcriptional profiling experiments.

GeneChip studies of the transcriptomes of laboratory grown the bacterial outer membrane. This finding prompted the authors to investigate whether UTI89 modulated iron acquisition genes during the transition from commensal to pathogen. Owing to their intracellular location, detecting gene expression in IBCs posed experimental challenges that were partly overcome by colonizing a mouse strain that lacked a functional Toll-like receptor 4. This strain has an impaired innate immune system and accumulates numerous IBCs that can be directly retrieved from host cells by laser capture microdissection.

Using quantitative RT-

PCR, Reigstad et al. showed that genes coding for siderophore synthetases, transporters and regulators, and proteins that scavenge haem-associated ferric iron sources, were significantly upregulated in laser captured, IBC-associated UTI89 compared with caecal UTI89. A mainstay of mammalian host defences is the ability to restrict iron availability for pathogens. Consistent with this, genes encoding transferrin (the mammalian iron-binding protein) and lipocalin 2 (which sequesters enterobactin) were upregulated in dissected-out bladder epithelial cells near to IBCs. Surprisingly, in immune-competent mice, of all the iron-acquisition loci that were upregulated in IBCs, only deletion of chuA in UTI89, which encodes the hemin receptor, reduced the size of IBCs.

Targeted functional genomics revealed that UPEC vies with the host for scant iron resources. These approaches should enable the battle that is waged in the host during the transition of UPEC from friend to foe to be more completely understood.

Susan Jones

ORIGINAL RESEARCH PAPER Reigstad, C. S. Hultgren, S. J. \& Gordon, J. I. Functional genomic studies of uropathogenic E. coli and host urothelial cells when intracellular bacterial communities are assembled. J. Biol. Chem. 14 May 2007 (doi:10.1074/jbc.M611502200). 